\title{
Tocilizumab, Remdesivir and Favipiravir repurposed for COVID-19: The Good, The Bad and The Ugly.
}

\author{
Mina Kelleni ${ }^{1}$ \\ ${ }^{1}$ Affiliation not available
}

November 30, 2020

Tocilizumab, Remdesivir and Favipiravir repurposed for COVID-19: The Good, The Bad and The Ugly.

Mina T. Kelleni, MD, PhD

Assistant Professor of Pharmacology, College of Medicine, Minia University, Egypt

Mobile: +201200382422 ,

email: mina.kelleni@mu.edu.eg, drthabetpharm@yahoo.com

https://orcid.org/0000-0001-6290-6025

Abstract:

In this manuscript, we compare the expectations versus the real word results and handling of three repurposed COVID-19 drugs; tocilizumab, remdesivir and favipiravir providing a personal perspective to affiliate a wellknown metaphor to describe their outcomes; the good, the bad and the ugly, respectively and encouraging those colleagues who consider these drugs in their COVID-19 management to consider the risk benefit ratio accordingly.

Keywords: COVID-19, Tocilizumab, Remdesivir, Favipiravir

Key summary points:

Tocilizumab might still have a potential to treat selected cases of COVID-19 though results of phase III clinical trials have been less than expected

Remdesivir, though its FDA approval and relatively high price, is very unlikely to significantly benefit COVID-19 patients

Favipiravir is least likely to safely manage COVID-19 patients and it's potentially teratogenic if given to pregnant patients

Drug repurposing is considered as a rapid strategy for COVID-19 drug discovery (Singh, Parida, Lingaraju, Kesavan, Kumar \& Singh, 2020) and many drugs have been tried. Tocilizumab, a recombinant humanized anti-IL-6 receptor monoclonal antibody FDA approved drug for diseases other than COVID-19, was initially shown to shorten the median time to COVID-19 clinical improvement (Kewan, Covut, Al-Jaghbeer, Rose, Gopalakrishna \& Akbik, 2020) and it also showed non-statistically significant lower mortality in patients with severe to critical COVID-19(Rojas-Marte et al., 2020). Interestingly, though several studies have even showed tocilizumab to significantly improve the clinical outcomes in severe cases of COVID-19 pneumonia (Alattar et al., 2020; Keske et al., 2020; Klopfenstein et al., 2020; Samaee, Mohsenzadegan, Ala, Maroufi \& 
Moradimajd, 2020; Toniati et al., 2020), yet phase III double blind, placebo-controlled clinical trials have recently declared that tocilizumab did not improve the clinical status in patients with COVID-19 associated pneumonia, and didn't prevent intubation or death in moderately ill hospitalized patients with Covid-19, though potential benefit/harm could not be entirely excluded (Roche, 2020, July 29; Stone et al., 2020). In comparison, some preliminary results anticipated the success of remdesivir, a nucleotide analogue prodrug, to treat COVID-19 (Ko et al., 2020; Lamb, 2020). However, other studies have suggested that remdesivir is unexpected to possess significant clinical benefits based on its released results and safety profile (2020; Carothers, Birrer \& Vo, 2020; Kelleni M, 2020, May 29; Kelleni, 2020, July 01; Marcolino, Pimentel \& Barão, 2020). Moreover, even open label phase III trial results showed no significant difference between a 10-day remdesivir course (median length of treatment, 6 days) compared with standard care while claiming a benefit for the 5-day course(Spinner et al., 2020), yet the drug continues to be marketed globally and priced at 3,120 USD for the typical patient with private insurance in USA. Moreover, a black market has developed in India (The Economic Times (Krishna Kumar), 2020, July 08) and perhaps other developing countries. Notably, the WHO Solidarity Trial Consortium has recently announced the results of randomized trials performed in 405 hospitals located in 30 countries where 2750 patients received remdesivir and it was shown to have little or no effect on hospitalized COVID-19 patients, as indicated by overall mortality, initiation of ventilation and duration of hospital stay(Pan et al., 2020) and despite all criticism and warnings, the FDA has recently approved remdesivir to be used for COVID-19(FDA, 2020, October 22) after an unprecedented consultation of a diverse group of experts outside the FDA (Jon Cohen, 2020, October 28). Fortunately, the WHO has declined to acknowledge remdesivir and recommended against its use in COVID-19 regardless of disease severity(WHO, 2020, November 20).

Similar to the remdesivir paradox, favipiravir (T-705) was approved in Japan in 2014 as a treatment for novel or re-emerging influenza viruses, its local approval was heavily restricted as its clinical use needed governmental authorization, which was only permitted for potentially lethal diseases and a similar cautious attitude was also adopted in China(Shiraki \& Daikoku, 2020). Furthermore, the Japanese drug safety bureau, possibly due to limited safety data, recommended favipiravir to be avoided where alternative drugs could be used and adverse effects such as hyperuricemia and teratogenicity/embryotoxicity were reported from favipiravir human and four different animal species studies, respectively and QTc prolongation potential was recommended to be further investigated (Pilkington, Pepperrell \& Hill, 2020; Shiraki \& Daikoku, 2020). Hepatitis was also reported in 4 out of 63 COVID-19 patients who received favipiravir(Rattanaumpawan, Jirajariyavej, Lerdlamyong, Palavutitotai \& Saiyarin, 2020). Notably, $>85 \%$ of favipiravir completed clinical trials in registries or in published papers are unavailable which led to failure of conducting meta-analysis to generate conclusive evidence to support recommendation of favipiravir use in COVID-19 or know about its safety and efficacy profile (Khambholja \& Asudani, 2020), and a Japanese study showed no significant efficacy of favipiravir in SARS CoV-2 elimination. This study also shows the cautious attitude of a Japanese health authority, Fujita Health University, which conducted the study as out of 47 facilities across Japan that took part; only 88 patients were enrolled and completed the study(The Mainichi, 2020, July 10). Most importantly, favipiravir is a nucleoside analogue that acts as a mutagen with a distinct bias to induce transitions in influenza virus RNAs by incorporating into both positive and negative stranded RNA and being aberrantly copied as multiple bases(Goldhill et al., 2019) and favipriavir-induced lethal mutagenesis of influenza virus may be the reason for the difficulty in isolating favipiravir-resistant influenza viruses in laboratories or to evolve resistance against clinically(Goldhill et al., 2019; Shiraki \& Daikoku, 2020).

In conclusion, we would like to recommend against the use of remdesivir without being proven by credible double blinded, placebo-controlled phase III clinical trials data that shows a real efficacy and safety of the drug which are extremely unlikely. Furthermore, a teratogenic, embryotoxic and virally mutagenic favipiravir should undergo extensive in vitro assays for potential gene mutation and/or chromosomal damage as well as long-term in vivo animal studies to evaluate its potential human carcinogenicity before allowing wide scale global trialing. On the other hand, we would also like to continue considering tocilizumab for critical cases of COVID-19 after considering the risk benefit ratio because of an overwhelming evidence coming from several independent and well-designed clinical trials and a remaining potential benefit for some cases that couldn't 
be excluded by a phase III clinical trial.

Conflict of interests:

None

Funding/Financial disclosure:

None

Acknowledgement

This main bulk of this manuscript has been denied, for months, an opportunity to be properly peer reviewed by several journals, however a draft has been sent by one of them to the WHO before its historic decision regarding remdesivir.

References:

(2020). Remdesivir-related acute kidney injury in patients with COVID-19. Reactions Weekly 1826: 5-5.

Alattar R, Ibrahim TBH, Shaar SH, Abdalla S, Shukri K, Daghfal JN, et al. (2020). Tocilizumab for the treatment of severe coronavirus disease 2019. Journal of medical virology: 10.1002/jmv.25964.

Carothers C, Birrer K, \& Vo M (2020). Acetylcysteine for the Treatment of Suspected Remdesivir-Associated Acute Liver Failure in COVID-19: A Case Series. Pharmacotherapy: The Journal of Human Pharmacology and Drug Therapy n/a.

FDA Approves First Treatment for COVID-19. [Online] Available from https://www.fda.gov/newsevents/press-announcements/fda-approves-first-treatment-covid-19. [Accessed].

Goldhill DH, Langat P, Xie H, Galiano M, Miah S, Kellam P, et al.(2019). Determining the Mutation Bias of Favipiravir in Influenza Virus Using Next-Generation Sequencing. Journal of virology 93:e01217-01218.

Jon Cohen KK (2020, October 28). The 'very, very bad look' of remdesivir, the first FDA-approved COVID-19 drug. In Science.

Kelleni M (2020, May 29). Rapid Response: Remdesivir is least likely to be effective for safe treatment of COVID-19: A pharmacovigilant point of view. BMJ 369: m2097rr.

Kelleni MT (2020, July 01). Remdesivir-Gate for COVID-19. Acta Scientific Gastrointestinal Disorders 3: 01.

Keske Ş, Tekin S, Sait B, İrkören P, Kapmaz M, Çimen C, et al.(2020). Appropriate use of tocilizumab in COVID-19 infection. International Journal of Infectious Diseases 99: 338-343.

Kewan T, Covut F, Al-Jaghbeer MJ, Rose L, Gopalakrishna KV, \& Akbik B (2020). Tocilizumab for treatment of patients with severe COVID-19: A retrospective cohort study. EClinicalMedicine 24: 100418.

Khambholja K, \& Asudani D (2020). Potential repurposing of Favipiravir in COVID-19 outbreak based on current evidence. Travel medicine and infectious disease 35: 101710-101710.

Klopfenstein T, Zayet S, Lohse A, Selles P, Zahra H, Kadiane-Oussou NdJ, et al. (2020). Impact of tocilizumab on mortality and/or invasive mechanical ventilation requirement in a cohort of 206 COVID-19 patients. International Journal of Infectious Diseases 99:491-495.

Ko W-C, Rolain J-M, Lee N-Y, Chen P-L, Huang C-T, Lee P-I, et al.(2020). Arguments in favour of remdesivir for treating SARS-CoV-2 infections. International journal of antimicrobial agents 55:105933105933.

Lamb YN (2020). Remdesivir: First Approval. Drugs 80:1355-1363. 
Marcolino VA, Pimentel TC, \& Barão CE (2020). What to expect from different drugs used in the treatment of COVID-19: A study on applications and in vivo and in vitro results. European Journal of Pharmacology 887: 173467 .

Pan H, Peto R, Abdool Karim Q, Alejandria M, Henao Restrepo AM, Hernandez Garcia C, et al. (2020). Repurposed antiviral drugs for COVID-19; interim WHO SOLIDARITY trial results. medRxiv:2020.2010.2015.20209817.

Pilkington V, Pepperrell T, \& Hill A (2020). A review of the safety of favipiravir - a potential treatment in the COVID-19 pandemic? J Virus Erad 6: 45-51.

Rattanaumpawan P, Jirajariyavej S, Lerdlamyong K, Palavutitotai N, \& Saiyarin J (2020). Real-world Experience with Favipiravir for Treatment of COVID-19 in Thailand: Results from a Multi-center Observational Study. medRxiv: 2020.2006.2024.20133249.

Roche provides an update on the phase III COVACTA trial of Actemra/RoActemra in hospitalised patients with severe COVID-19 associated pneumonia. [Online] Available from https://www.roche.com/investors/updates/inv-update-2020-07-29.htm. [Accessed].

Rojas-Marte G, Khalid M, Mukhtar O, Hashmi AT, Waheed MA, Ehrlich S, et al. (2020). Outcomes in patients with severe COVID-19 disease treated with tocilizumab: a case-controlled study. QJM 113: 546-550.

Samaee H, Mohsenzadegan M, Ala S, Maroufi SS, \& Moradimajd P (2020). Tocilizumab for treatment patients with COVID-19: Recommended medication for novel disease. International Immunopharmacology 89: 107018.

Shiraki K, \& Daikoku T (2020). Favipiravir, an anti-influenza drug against life-threatening RNA virus infections. Pharmacology \& Therapeutics 209: 107512.

Singh TU, Parida S, Lingaraju MC, Kesavan M, Kumar D, \& Singh RK (2020). Drug repurposing approach to fight COVID-19. Pharmacological Reports.

Spinner CD, Gottlieb RL, Criner GJ, Arribas López JR, Cattelan AM, Soriano Viladomiu A, et al. (2020). Effect of Remdesivir vs Standard Care on Clinical Status at 11 Days in Patients With Moderate COVID-19: A Randomized Clinical Trial. JAMA 324: 1048-1057.

Stone JH, Frigault MJ, Serling-Boyd NJ, Fernandes AD, Harvey L, Foulkes AS, et al. (2020). Efficacy of Tocilizumab in Patients Hospitalized with Covid-19. New England Journal of Medicine.

Remdesivir sold at high prices in Mumbai black market; some doctors suggest cheaper alternatives [Online] Available from https://economictimes.indiatimes.com/industry/healthcare/biotech/pharmaceuticals/remdesivirsold-at-high-prices-in-mumbai-black-market-some-doctors-suggest-cheaper-alternatives/articleshow/76861773.cms?utm_source $=$ contentofinterest\&utm_medium $=$ text\&utm_campaign $=$ cppst. [Accessed].

Avigan study fails to demonstrate benefit in COVID-19 treatment.[Online] Available from https://mainichi.jp/english/articles/20200710/p2g/00m/0fe/107000c. [Accessed].

Toniati P, Piva S, Cattalini M, Garrafa E, Regola F, Castelli F, et al. (2020). Tocilizumab for the treatment of severe COVID-19 pneumonia with hyperinflammatory syndrome and acute respiratory failure: A single center study of 100 patients in Brescia, Italy. Autoimmunity Reviews 19: 102568.

WHO (2020, November 20). WHO recommends against the use of remdesivir in COVID-19 patients. 